\title{
Overview of Cardiac Rehabilitation Evidence, Benefits and Utilisation
}

\author{
Abdulrahman Al Quait ${ }^{1,2} \&$ Patrick Doherty ${ }^{1}$ \\ ${ }^{1}$ Department of Health Sciences, Faculty of Science, University of York, York, United Kingdome \\ ${ }^{2}$ King Fahad Medical City, Riyadh, Saudi Arabia \\ Correspondence: Abdulrahman Al Quait, Department of Health Sciences, Seebohm Rowntree Building, Area 4, \\ University of York, UK. Tel: 44-(0)-190-432-1103. E-mail: aiaq500@york.ac.uk
}

Received: September 8, 2017 Accepted: October 10, 2017 Online Published: Decmber 19, 2017

doi:10.5539/gjhs.v10n2p38 URL: https://doi.org/10.5539/gjhs.v10n2p38

\begin{abstract}
Historically, the main objective of cardiac rehabilitation (CR) as an exercise-based programme was to restore or improve patients' regular physical activity after a cardiac event. Since then CR has evolved into a comprehensive secondary prevention programme, the objectives of $\mathrm{CR}$, and indications and contraindications for its use have also developed in sophistication. Current CR programmes are designed to stabilise or even reverse the progression of heart disease by controlling all modifiable risk factors. They are also concerned with improving patients' quality of life by restoring their wellbeing. All this should be achieved with the maximum safety levels to patients. The first part of this review details on how CR evolved from a simple exercise programme to a comprehensive secondary prevention programme in the past few decades. The second part sets an example of modern CR provision, pathway and guidelines in a top leading country in this field, the UK.
\end{abstract}

Keywords: cardiac rehabilitation, secondary prevention, coronary artery disease

\section{Introduction}

In medicine, while saving a patient's life is clearly vital, it is also important to attempt to help patients recover a good quality of life. Experiencing a heart attack or a hospital admission following a cardiac event can be very stressful and patients who suffer such a dramatic event will definitely need support to live with their new heart condition. In particular, they will need help to stay as healthy as possible and therefore reduce the risk of suffering another event. Knowing that coronary artery disease (CAD) is a long term and progressive condition, the concept of rehabilitation in cardiac care is that the recipients gain the knowledge and support necessary to live as normal a life as possible alongside their cardiac condition.

Cardiac rehabilitation (CR) has been defined by various organisations and national entities and can be encompassed by:

"The coordinated sum of activities required to influence favourably the underlying cause of cardiovascular disease, as well as to provide the best possible physical, mental and social conditions, so that the patients may, by their own efforts, preserve or resume optimal functioning in their community and through improved health behaviour, slow or reverse progression of disease" (BACPR, 2017).

Traditionally, exercise training is known as the core component of CR. Current practices guidelines, however, push towards "comprehensive rehabilitation" and thus CR's regulating bodies drive programmes to add other components to optimise the reduction of risk factors and improve adherence to healthy behaviours among recipients. Those components include health education, advice on the reduction of cardiac risk factors and stress management.

\subsection{Historical Background to Cardiac Rehabilitation}

For centuries, bed rest was considered essential in the treatment of a disease, and mobility restrictions were imposed on most patients, and particularly patients with heart disease. This practice was not challenged until 1802 when Heberden reported the observation of an angina patient who improved after sawing wood for half an hour a day (Didier, Bigand, Vinter, \& Boucheix, 2010). Despite some evidence of the benefits of physical activity for heart patients, the misguided focus on bed rest persisted until chair therapy was introduced in the 1940s. A few years later, daily short walks of 3 to 5 minutes were allowed four weeks after the coronary event (Mampuya, 2012). Since then advances in clinical research have led to better ways of understanding heart disease management. 
The accumulation of evidence of the benefits for cardiac patients of early physical activity started in 1953 when a study conducted by Morris and Heady showed that CHD was at least one third higher in light occupation workers compared to heavy occupation workers (MORRIS \& HEADY, 1953). The decrease in CHD incidents was attributed to the extra physical activity performed by heavy occupation workers. In 1966, Saltin and co-workers conducted a study on five men, each 20 years of age, to compare the effects of three weeks of bed rest and eight weeks of intense physical exercise on the subjects' physical condition. Regardless of its small sample size, this study, which was known as the 'Dallas Bed Rest and Training Study', enriched the early discussions and analyses of the positive impact of exercise and the detrimental effects of bed rest on subjects' physical condition (McGavock et al., 2009).

In the late 1970s, the concept of prescribing exercise therapy for post-MI patients began to gain momentum as it became increasingly clear that immobilisation and reduced activity results in poor long-term prognosis and survival (Lear \& Ignaszewski, 2001). The establishment of the benefits of exercise therapy on patients' prognosis led to a surge of interest in the effects of exercise therapy on morbidity and mortality rates which then revealed undeniable improvements. Since that time a large and growing body of literature has emerged to establish the physiologic basis of the benefits of exercise and this has led to the development of CR programmes.

Nowadays, CR is a complex intervention that has eventually evolved from the emphasis on exercise therapy to become a comprehensive secondary prevention programme that provides patients with supervised exercise and education sessions to help them to recover and get back to as full a life as possible. CR has been proven to be as much a part of CAD treatment as medicines are. Unfortunately, the proven benefits of CR are not matched by a strong endorsement of $\mathrm{CR}$ in the cardiology community. The reason behind this reticence can be attributed to the development of powerful drugs and new therapeutic technologies that have made it difficult for CR to compete in its own right, since cardiologists are apparently attracted to the immediate and short-term results of these conventional direct medical interventions. Recent years, however, have seen increasingly rapid advances in the field of cardiology. These recent developments have led to a better understanding of heart disease management which is in turn reflected in a renewed interest in CR. Hopefully, the coming years will witness a rapid rise in CR utilisation rates.

\section{Modern Cardiac Rehabilitation}

Historically, the main objective of $\mathrm{CR}$ as an exercise-based programme was to improve patients' regular physical activity after a cardiac event (Mampuya, 2012). Since CR has evolved into a comprehensive secondary prevention programme, the objectives of $\mathrm{CR}$, and indications and contraindications for its use have also developed in sophistication. Current CR programmes are designed to stabilise or even reverse the progression of heart disease by controlling all modifiable risk factors. They are also concerned with improving patients' quality of life by restoring their wellbeing. All this should be achieved with the maximum safety levels to patients.

\subsection{Objectives}

The main objective of CR continues to be helping recipients to regain their autonomy by improving regular physical activity after a cardiac event. Controlling the modifiable risk factors and therefore reducing the negative effects of CAD is another objective of CR. The term risk factor is defined by WHO as:

"any attribute, characteristic or exposure of an individual that increases the likelihood of developing a disease or injury" (WHO, 2014).

Obviously, the more risk factors a subject has for a specific disease, the greater the chance they have of acquiring that disease. In cardiology, there are modifiable risk factors (those that can be controlled) and unmodifiable risk factors (which cannot be changed). Unmodifiable risk factors for CAD include age, gender, ethnicity and family history. Although unmodifiable risk factors cannot be changed, making changes to patients' lifestyles can significantly reduce the risk of acquiring CAD prematurely. Other characteristics that may lead to an increased chance of acquiring CAD include social, economic and cultural change (e.g. urbanisation). Stress and poverty are also other determinants of an increased chance of getting CAD (WHO, 2014). These characteristics and determinants are not direct causes of CAD but can be looked at as "the causes of the causes". Modifiable risk factors that may have an adverse impact on the prognosis of CAD include: hypertension, hypercholesterolemia, diabetes, sedentary lifestyle, obesity and smoking (Montalescot et al., 2013). Curing or at least controlling these factors can significantly reduce the progression of CAD disease.

A comprehensive CR programme should also educate recipients about their conditions so as to allow them to become responsible for their medical treatment and lifestyle changes and therefore achieve optimal outcomes (Mampuya, 2012). These education sessions should be delivered in a structured fashion for instance using 
workshop-based teaching programmes delivered and by a skilled and experienced multidisciplinary team such as dietitians, psychologists, exercise specialists, etc. (BACPR, 2017). Anxiety and depression, for example, have been reported to be associated with lower exercise capacity, fatigue and sense of wellbeing (Mampuya, 2012). The use of a skilled and experienced psychiatrist to educate recipients about stress management and self-control tools can help recipients to have a better control of other risk factors.

Lastly, CR programmes should aim to limit the physiological and psychological effects of heart disease by controlling disease symptoms and the side effects of medications. This should have a favourable impact on patients' quality of life, making the benefits of CR more tangible to recipients and therefore encouraging them to complete the programme, foster healthy behaviours and thus achieve optimal outcomes (Dalal, Doherty, \& Taylor, 2015).

\subsection{Indications}

The literature on CR has highlighted several patient groups that should benefit from joining a CR programme. Generally accepted indications for CR include: myocardial infarction (MI), coronary artery bypass graft (CABG), percutaneous coronary intervention (PCI), valve repair or replacement and angina. The recent trends in CR which encourage programme commissioners to tailor special programmes for special patient groups have widened the scope of CR to include patients with heart failure and heart transplants. Different countries allocate different resources into CR, however, and therefore the indications for CR may vary between countries. In the UK, the National Institute for Health and Care Excellence (NICE), Department of Health, British Association for Cardiovascular Prevention and Rehabilitation (BACPR), and wider European guidelines agree that the patient groups listed in (Table 1) will benefit from a CR programme (Dalal et al., 2015).

Table 1. Patient groups who benefit from CR (Note 1)

\begin{tabular}{ll}
\hline Indication & Description \\
\hline ACS & $\begin{array}{l}\text { Including STEMI, NSTEMI, and unstable angina; also all patients undergoing } \\
\text { reperfusion (such as CABG, primary PCI and elective PCI). }\end{array}$ \\
Heart Failure (HF) & $\begin{array}{l}\text { Patients with newly diagnosed chronic HF and chronic HF with a step change in clinical } \\
\text { presentation. }\end{array}$ \\
Heart surgery & $\begin{array}{l}\text { Heart transplant, ventricular assist device, intra-cardiac defibrillator, valve replacement or } \\
\text { repair and cardiac resynchronisation therapy. }\end{array}$ \\
Angina & Patients with a confirmed diagnosis of exertional angina. \\
\hline
\end{tabular}

\subsection{Contraindications \& Safety}

Contraindications for the use of $\mathrm{CR}$ are only concerned with the exercise aspect of the programme while all other parts of CR are considered relatively safe. Since the exercise in a CR programme is medically prescribed and supervised, the risks of $\mathrm{CR}$ are considered to be low, and most patients referred to $\mathrm{CR}$ are eligible to participate in the programme. Furthermore, the capacity of CR providers to tailor their programme to suite the medical needs of individual patients has reduced the contraindicated patient groups even further. Nonetheless, medically unstable or life threatening conditions are examples of patients who are not eligible to enrol in a CR programme (Thomas et al., 2010).

The literature on CR has highlighted a number of studies that have documented the safety of exercise in a CR programme. In 2007, the Americac Heart Association (AHA) issued a statement to discuss the potential complications of exercise in a CR programme. This statement has estimated the incidence of exercise-related cardiovascular complications in CHD patients as one cardiac arrest per 116,906 patient-hours of supervised exercise, one MI per 219,970 patient-hours of supervised exercise, one death per 752,365 patient-hours of supervised exercise and one major complication per 81,670 patient-hours of supervised exercise (P. D. Thompson et al., 2007).

Another observational study from a French registry with a population of 25,000 patients has reported one cardiac incident per 50,000 hours of supervised exercise. This is equivalent to 1.3 cardiac arrests per million patient-hours (Pavy, Iliou, Meurin, Tabet, \& Corone, 2006). A qualitative Japanese study surveyed 136 hospitals operating core phase CR amounting to 383,096 patient-hours of supervised exercise. The incident rate of all adverse events was 12 per 383,096 patient-hours while life-threatening events (death, cardiac arrest, AMI and cardiac rupture) occurred at the rate of one per 383,096 patient-hours of supervised exercise. This is equivalent to 3.13 and 0.26 
events per 100,000 patient-hours of supervised exercise respectively.

Taken together, these results suggest that supervised exercise in a CR programme is generally safe. These findings can only be extended to CR programmes that are equipped to handle major emergency events such as cardiac arrest, however. In addition, it should be noted that subjects in the above studies are medically evaluated before enrolling in the programme which could decrease the number of incident rates. In general, all these considerations support safety in supervised exercise programmes but they should not be extrapolated to home based programmes.

\subsection{Cardiac Rehabilitation Benefits}

The benefits of CR for the indicated patient groups have been reviewed comprehensively in several systematic reviews and meta-analyses including seven Cochrane reviews. However, these benefits remain controversial in the modern era of statins and acute revascularisation. A recent systematic review and met-analysis of randomised and non-randomised studies has sought to assess the actual evidence of CR in the modern era of cardiology, the Cardiac Rehabilitation Outcome Study (CROS) review(Rauch et al., 2016). These benefits are the result of all components of a comprehensive multi-disciplinary CR programme. In this section, the benefits of a comprehensive CR programme will be outlined with brief supporting evidence from the literature.

\subsubsection{Mortality}

A 2016 Cochrane review and meta-analysis of 63 randomised control trials (RCT) with 14,486 participants with a median follow-up of 12 months showed that $\mathrm{CR}$ has a varied effect on mortality. In this review 47 trials with a total sample size of 12,455 subjects reported all-cause mortality. Reduction in all-cause mortality between control and intervention groups was not statistically significant in these studies (relative risk (RR): 0.96 ; $95 \%$ confidence interval (CI): 0.88 to 1.04$)$. On the other hand, 27 trials $(n=7,469)$ reported cardiovascular mortality and showed a statistically significant reduction of cardiovascular mortality (RR: $0.74 ; 95 \%$ CI: 0.64 to 0.86 ). Twenty studies in this review reported both types of mortality and results in this sub-group were consistent with the overall meta-analysis results (all-cause mortality RR: $0.91,95 \%$ CI: 0.82 to 1.01 ; cardiovascular mortality RR: $0.78,95 \%$ CI: 0.67 to 0.90) (Anderson et al., 2016).

Another systematic review and meta-analysis of 34 RCT's with a total pooled population of 6,111 subjects has revealed a reduction in all-cause mortality for CR patients compared to control groups by $26 \%$ (odds ratio (OR): $0.74,95 \%$ CI: 0.58 to 0.95 ). It also showed a reduction in cardiovascular mortality by $36 \%$ (OR: $0.64,95 \%$ CI: 0.46-0.88) (Lawler, Filion, \& Eisenberg, 2011). Conversely, a UK based trial titled 'Rehabilitation after myocardial infarction trial (RAMIT)' reported no significant difference in mortality between patients referred to rehabilitation and controls at two years follow up period (RR: $0.98,95 \%$ CI: 0.74 to 1.30 ) or after 7-9years (RR: $0.99,95 \%$ CI: 0.85 to 1.15 ) (West, Jones, \& Henderson, 2012). The negative findings of this trial can be attributed to the biased study design and failure to recruit a large enough sample size for the trial $(<1000$ patient in each arm of trial). In fact, RAMIT was designed to measure the effectiveness of CR as provided in 'real life' rather than if CR 'works' (Doherty \& Lewin, 2012).

This section has attempted to provide a brief summary of the literature relating to the impact of CR on all-cause mortality and cardiovascular mortality. In general, it seems that enrolling in a CR programme can impact rates of mortality favourably. The inconsistency in the literature may be due to several reasons related to the included studies' design such as study periods and population, duration of CR, intensity of CR or whether CR was delivered effectively.

\subsubsection{Hospital Admissions}

To date, the latest Cochrane systematic review has shown that the risk of hospital admission was reduced by $18 \%$ when comparing exercised-based CR with usual care (RR: 0.82; 95\% CI: 0.70 to 0.96) (Anderson et al., 2016). This data is based on 15 RCTs with a population size of 3,030 patients. Another systematic review aiming to update a previously published Cochrane systematic review of exercise-based CR for heart failure patients, measured hospital admission as a CR outcome. This study systematically reviewed 33 trials with six or more months of follow-up and included only patients with reduced ejection fraction $(<40 \%)$ and New York Heart Association class II and III $(\mathrm{n}=4,740)$. The findings revealed a reduced risk of overall hospital admission (RR: 0.75 ; $95 \%$ CI: 0.62 to 0.92 ) and heart failure-specific admission (RR: $0.61 ; 95 \%$ CI: 0.46 to 0.80 ) in patients who received comprehensive CR compared with no exercise controls (Sagar et al., 2015). A drawback of this study, in addition to the inclusion of old studies, was the inclusion of trials with relatively small sample sizes and short-term follow-up. This reflected on the number of hospital admissions reported by the majority of the included trials.

The main two challenges in modern cardiology are cost and capacity. It can therefore be assumed that CR, by reducing the number of hospital admissions, is a significant tool in facing the challenges in modern cardiology. It 
can thus also be said that the outcomes of CR go beyond the benefits to individuals and encompass improvements to the whole cardiac care system.

\subsubsection{Quality of Life and Psychological Wellbeing}

Two of the main objectives of a comprehensive CR programme are to improve the quality of life and the psychological state of the patient. Improvements in quality of life can be achieved through improvement in exercise performance (fitness) and through alleviation of symptoms, i.e. reducing chest pain, dyspnoea and fatigue (Mampuya, 2012). A Cochrane review of exercise-based CR for CHD patients reported 20 trials, with a total population of 5,060 subjects, assessing Health Related Quality of Life (HRQL) using a variety of validated generic or disease-specific outcome measures. Fourteen of the 20 trials $(65 \%)$ documented an increase in HRQL in one or more domains in patients subsequent to a CR programme compared to controls. Within these 14 trials, five reported a higher level of HRQL in at least one-half of the subscales (Anderson et al., 2016). The authors of the Cochrane review, however, expressed the HRQL scores as mean differences and were unable to quantify the effect of the 14 trials together due to the heterogeneity among the included studies.

This view is supported by another Cochrane systematic review that was conducted on heart failure patients. Sagar et al. (2015) reviewed a total of 18 trials which reported a validated HRQL measure. Thirteen of those 18 trials (72\%) reported higher HRQL scores in patients following exercised-based CR programmes compared with control subjects (Sagar et al., 2015). This finding provides a valuable insight into the subject as all 13 trials used the same validated HRQL scoring measure, the disease-specific Minnesota Living with Heart Failure Questionnaire (MLHFQ).

Another benefit of $\mathrm{CR}$ is to improve the psychological state of the patient by stress reduction and the enhancement of the overall sense of psychosocial wellbeing. A meta-analysis of 23 RCTs with a total population size of 3,180 CAD patients was conducted to evaluate the impact of including a psychosocial component within a standard exercise-based CR programme. Patients who received psychosocial intervention showed greater reductions in psychological distress (with effect size differences of 0.34) (Linden, Stossel, \& Maurice, 1996). An American observational study tried to assess improvements in depression in patients who developed heart failure due to CHD after receiving a comprehensive CR programme. Depressive symptoms were assessed at baseline and after CR by standard questionnaire (Kellner Symptom Questionnaire). In patients who completed CR $(n=151)$ depressive symptoms decreased by $40 \%$ post CR, from $22 \%$ to $13 \%(\mathrm{p}<0.0001)$. In addition, Depressed patients who completed CR had a $59 \%$ lower mortality $(44 \%$ vs $18 \%$, p < 0.05$)$ compared to depressed dropout subjects $(\mathrm{n}=38)$ (Milani, Lavie, Mehra, \& Ventura, 2011). This study, however, suffers from the limited sample size, especially in the dropout subjects and also from using a questionnaire that is not well established for measuring depressive symptoms in clinical trials of depression.

Furthermore, two observational studies, one British $(n=465,825)$ and one American $(n=635)$, reported improvements in anxiety and depression after CR (Abdulrahman Al Quait \& Doherty, 2016; Lavie \& Milani, 2006). Although the main objective of both studies was to compare levels of improvement in a range of outcomes between young and old patients, varied improvements in anxiety and depression were evident post CR in both groups with younger patients achieving better outcomes.

\subsubsection{Cardiovascular Risk Profile}

Modern CR programmes are designed to provide a reduction in the modifiable cardiovascular risk factors. A systematic review and meta-analysis of 34 RCT's $(n=6,111)$ examined the effect of CR on modifiable risk factors. Overall, trials found a more favourable effect on the prevalence of risk factors among subjects who were randomised to $\mathrm{CR}$ compared with controls. The risk factors in which improvements were observed in the intervention groups in these trials were smoking cessation, blood pressure and total cholesterol, whereas changes in body weight were minimal in both groups (Lawler et al., 2011).

Another systematic review and meta-analysis of 23 trials and a total population size of 3,180 (2,024 intervention subjects and 1,156 controls) CAD patients reported the impact of CR on selected modifiable risk factors. The findings revealed that patients who received CR showed a greater reduction in systolic blood pressure, heart rate and cholesterol level (with effect size differences of $-0.24,-0.38$, and -1.54 , respectively) (Linden et al., 1996). These results must be interpreted with caution, however, since the effect of medical drugs such as statins and beta-blockers could not be excluded.

In a large cohort retrospective observational study $(n=465,825)$ Al Quait \& Doherty conducted an evaluation of nine patient outcomes pre and post CR. The outcomes reported in this analysis were Body Mass Index (BMI), waist size, total cholesterol, blood pressure, smoking, walking fitness, physical activity, anxiety and depression. 
Although the primary objective of the study was to determine if CR outcomes were influenced by age in CHD patients, undeniable improvements were documented in modifiable risk factors in both groups. This analysis revealed that elderly patients achieved better outcomes in body shape risk factors while younger patients achieved much better outcomes across a wider range of risk factors, in particular with regards to smoking cessation (Abdulrahman Al Quait \& Doherty, 2016).

\section{Cardiac Rehabilitation in the UK}

The availability of CR and how it is organised varies across countries. This is mainly to do with differences in health policies and politics since different countries allocate different resources into the healthcare system. Resource allocation is largely dependent on the income of each country, with high income countries generally investing more in healthcare services, and therefore CR, than low income countries (Mampuya, 2012). Worldwide, the availability of CR is low compared to the benefits it offers, as only $38 \%$ of countries globally have CR programmes. More specifically, $68 \%$ of those programmes are allocated in high income countries, $28.2 \%$ in middle income countries and only $8.3 \%$ in low income countries (Turk-Adawi, Oldridge, Tarima, Stason, \& Shepard, 2013). The provision, pathway and guidelines of CR can therefore be different across countries.

\subsection{History of Cardiac Rehabilitation in the UK}

The earliest attempt to promote CR in the UK goes back to the year 1970 when Groden and co-authors circulated a questionnaire to all active adult cardiologists of the British Cardiac Society to assess their attitude towards CR. With a $75 \%$ response rate, the survey revealed that $74.3 \%$ of cardiologists were in favour of developing special services for the rehabilitation of cardiac patients whereas $25.7 \%$ felt that such special services were not indicated (Groden, Semple, \& Shaw, 1971). The same study, however, found just nine cardiologists were in a position to offer CR to their patients in a special CR centre. In 1989 the number of CR centres had risen to 99 and by the year 1992 the BHF could identify 151 centres (Bethell, Turner, EJ, \& Rose, 2000). This figure continues to rise and in the first NACR report in 2007, 312 UK programmes have been identified (NACR, 2016).

Until the introduction of the first guidelines and audit standards in 1996, there had been no protocol for the provision of CR in the UK and there was no information on what most programmes did, nor who their subjects were (Bethell et al., 2000). Two years prior to the introduction of the first national guidelines, specifically in January 1994, Thompson and colleagues posted a survey to the senior nurse of 244 centres in England and Wales to determine the level of $\mathrm{CR}$ provision. With a $100 \%$ response rate the results showed that most programmes were based in hospitals (92\%) and had been in operation for between three months and 17 years. Content-wise, $100 \%$ of the programmes were delivering education and supervised exercise sessions, $96 \%$ were delivering stress management sessions and 40\% counselling (D R Thompson, Bowman, Kitson, de Bono, \& Hopkins, 1996). In 1997, the BACPR, which was then known as BACR and had been established in 1993, embarked on a three stage questionnaire survey designed to evaluate the quality of CR provision in the UK, promote record keeping and to measure CR outcomes (Bethell et al., 2000). Since then, research into CR in the UK has evolved and significant funding has been attracted from sources including the BHF, the Department of Health and the Medical Research Council (David R. Thompson, 1998). This has resulted in placing the UK into the top $2 \%$ of countries in Europe in CR uptake (NACR, 2016).

In 2000, a target was set for England that, by 2002, 85\% of MI, PCI and CABG patients should be invited to attend CR (NACR, 2007). To ensure that this target is met and that all established guidelines are adhered to, the National Audit of Cardiac Rehabilitation (NACR) was formally established in the 2005, led by Prof Bob Lewin, and the first national audit report was published in 2007. In 2013 the NACR, under Prof Doherty's leadership and supported by the BHF, took a significant step in transforming the aduit into a service improvement and quality assurance system where the ultimate beneciaries are CVD patients. Nowadays, the overall mean uptake to CR in the UK has achieved a significant milestone by reaching $50 \%$. This improvement brings the UK into the top $2 \%$ of countries in Europe (NACR, 2016).

\subsection{Structure}

Traditionally, $\mathrm{CR}$ in the UK has been delivered by clinicians in supervised groups of patients in outpatient hospital clinics or community centres (Dalal et al., 2015). NICE clinical guidelines advise the National Health Service (NHS) on caring for people with specific conditions, such as heart disease, and the treatment they should receive, while the BACPR is an association that represents CR professionals in the UK and is responsible for setting the standards by which clinicians work. The NACR, funded by BHF and hosted by the University of York, collects comprehensive audit data to support the improvement and monitoring of CR services in terms of their uptake, quality and clinical outcomes. Since 2016, the BACPR works in conjunction with the NACR implemented a 
national certification system which ensures that CR programmes in the UK are meeting minimum standards. The structure of NACR in the UK is illustrated in Figure 1.

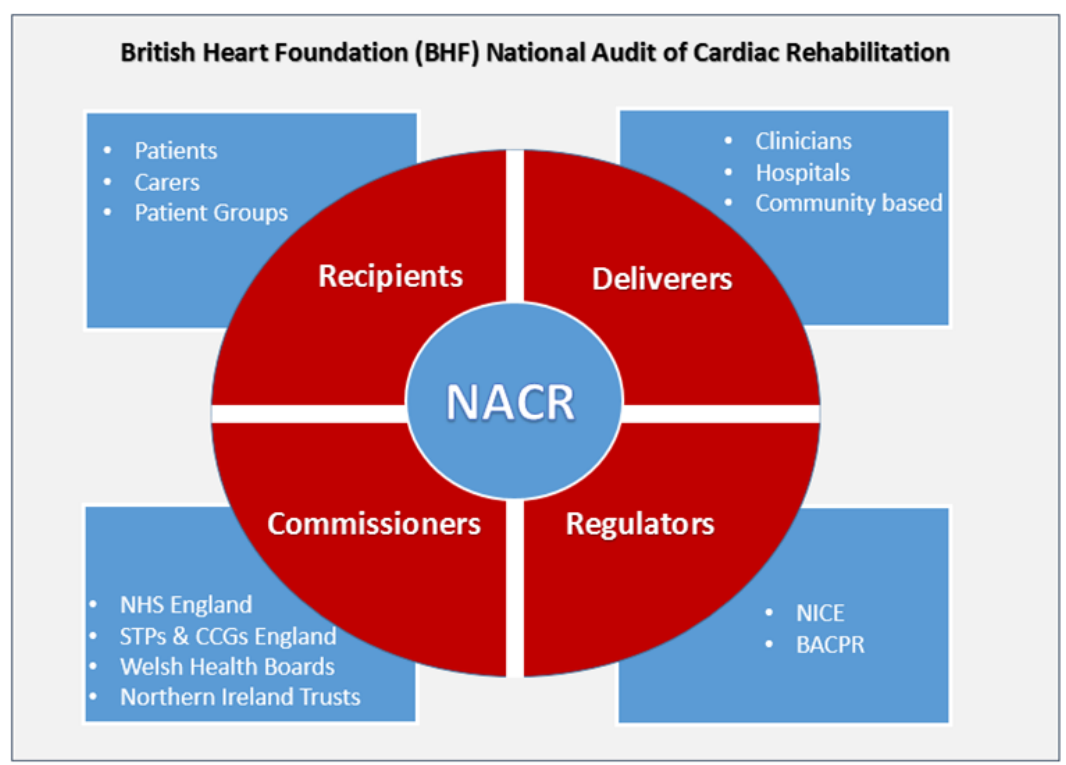

Figure 1. Structure of NACR in the UK (Note 2)

\subsection{Pathway}

Recent guidance from the UK Department of Health commissioning guide for CR refers to a six stage pathway of care that begins with patient presentation (diagnosis of a cardiac event) and is followed by identification for eligibility, referral and recruitment, baseline assessment and the development of a care plan, delivery of a comprehensive $\mathrm{CR}$ programme, the completion of a final CR assessment and then discharge and transition to long term management (Figure 2).

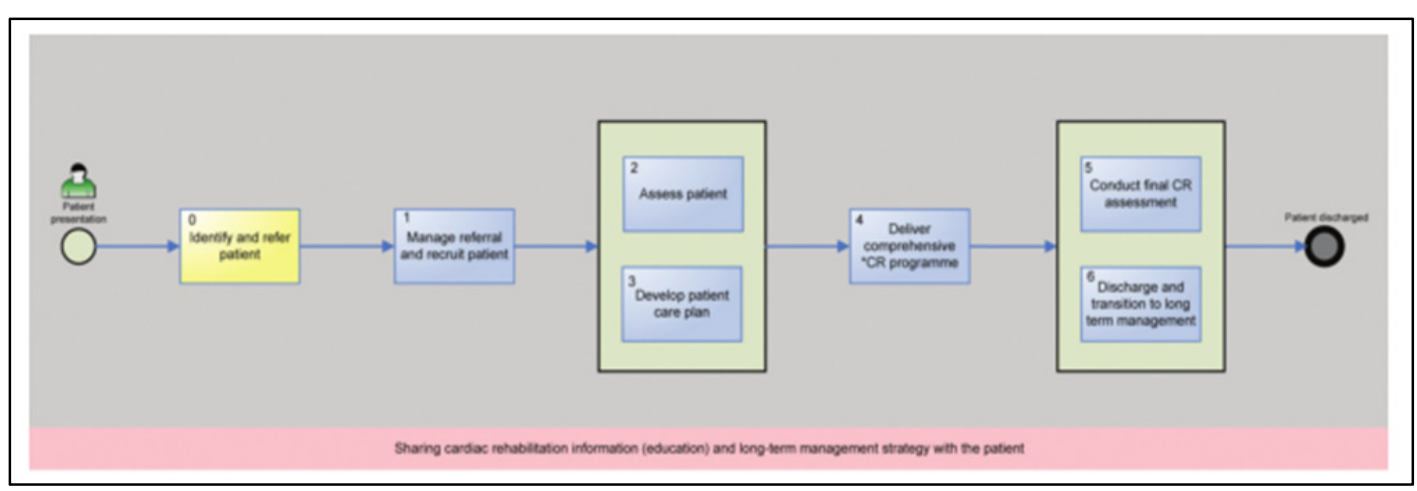

Figure 2. Department of Health Commissioning Guide Six-Stage Patient Pathway of Care (Note 3)

Each stage in this pathway is vital for CR uptake, adherence and outcomes and, overall, it should have an effect on long-term behavioural change leading to the desired health outcomes (BACPR, 2017).

\subsection{Standards}

Formal CR programmes vary between countries in terms of their delivery, intensity and duration. All programmes, however, are seeking the presentation of evidence-based best practice to provide effective prevention and rehabilitation services to their patients. As the association in charge of setting the standards by which CR clinicians work, BACPR continuously revises and updates CR standards in the UK.

In the updated third edition of the BACPR Standards and Core Components (2017), these standards and core components have been reduced from seven to six with the aim of this reduction being to increase the emphasis on 
measurable clinical outcomes, audit and certification (BACPR, 2017). The six standards for cardiovascular prevention and rehabilitation as issued by BACPR are:

Standard One The delivery of six core components by a qualified and competent multidisciplinary team, led by a clinical coordinator.

Standard Two Prompt identification, referral and recruitment of eligible patient populations.

Standard Three Early initial assessment of individual patient needs which informs the agreed personalised goals that are reviewed regularly.

Standard Four Early provision of a structured cardiovascular prevention and rehabilitation programme (CPRP), with a defined pathway of care, which meets the individual's goals and is aligned with patient preference and choice.

Standard Five Upon programme completion, a final assessment of individual patient needs and demonstration of sustainable health outcomes.

Standard Six Registration and submission of data to the National Audit for Cardiac Rehabilitation (NACR) and participation in the National Certification Programme (NCP_CR). (BACPR, 2017).

These revised standards and core components place health behaviour change and education at the very centre of CR programmes whilst placing equal emphasis on risk factor management and psychosocial health. Standard six, for instance, has been designed in order to ensure that CR programmes in the UK are meeting, or at least working towards, the minimum standards.

\subsection{Utilisation}

Existing research recognises the critical role played by CR in heart disease prevention. Despite the robust evidence of the clinical benefits and cost effectiveness of CR, utilisation rates vary widely worldwide, ranging from $20 \%$ to $50 \%$ (Dalal et al., 2015). Different healthcare policies and CR delivery systems among countries may explain, at least partially, this variability (Mampuya, 2012).

In the year 2000 England was set a target that, by $2002,85 \%$ of MI, PCI and CABG patients should be invited to attend CR. The first NACR report in 2007, however, revealed that this target was far from being met on the ground: of the 152,417 new eligible cardiac patients in the year April 2005 to March 2006, only 65,012 received CR, around $40 \%$ (NACR, 2007). A few years later, the NHS England CVD strategy for 2015/16 set a target to increase CR uptake to 65\% for patients admitted with CAD (NHS England Guideline 2014). The 2016 NACR report showed that the overall uptake of eligible patients in CR programs is only 50\%, a 3\% increase from 2015 and $5 \%$ increase from 2014 (NACR, 2016). Although current CR utilisation rates in the UK fall below national recommendations, however, they still far exceed those seen in other European countries, with an overall mean of $30 \%$ (Humphrey, Guazzi, \& Niebauer, 2014). In fact, with a 50\% average CR uptake in 2016, CR programmes in the UK are among the highest uptake figures globally (NACR, 2016).

To date, however, little research has been conducted in regards to CR utilisation in the growing PCI population and it is not clear what factors influence this. Figure 3 illustrates CR utilisation rates in PCI patients for the past six years in the UK.

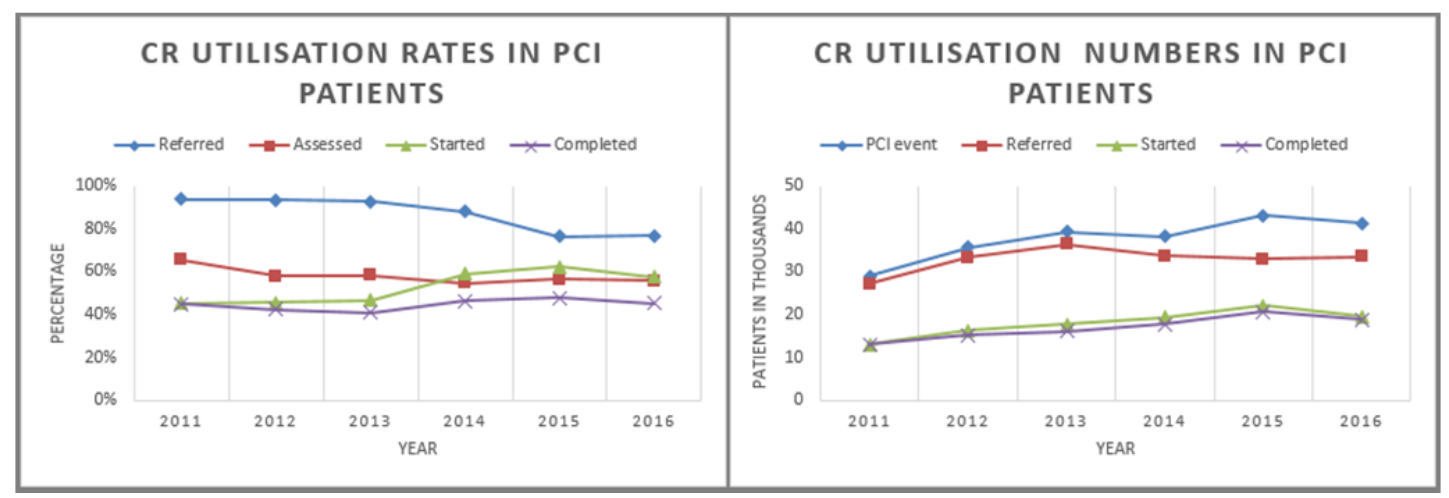

Figure 3. CR utilisation rates in PCI patients as measured by NACR 
The graph shows that there has been a gradual increase in the number of patients starting CR over the years although the number of referred patients dropped starting from the year 2013. Figure 3 also reveals that the number of PCI procedures has increased from around 28,000 in 2011 to more than 41,000 patients in $2016(46 \%)$. It also shows that the gap between those who start and those whom complete the programme is getting wider, however, this might be attributed in part to the fact that the quality of data entry in the NACR portal has improved in recent years.

When CR utilisation rates are analysed in respect to the type of treatment received, more gaps can be seen. The NACR 2016 report has shown low uptake rates in patients following MI (39\%) and elective PCI (45\%) compared to the world-leading uptake rate in the CABG population (60\%). This is of major concern when we realise that there were 92,445 PCI procedures carried out in the UK in 2012 compared to 16,791 CABG procedures (Townsend, Bhatnagar, Wilkins, Wickramasinghe, \& Rayner, 2015). In other words, around 50,000 eligible CR patients were not offered CR to prevent further MI and the progression of CAD (NACR, 2014). Although NHS England's CVD strategy for $2015 / 16$ has an ambition to increase the uptake of CR to $65 \%$ for patients admitted with CAD, only a $2 \%$ increase in uptake has been recorded from the previous year (England \& Guideline, 2014; NACR, 2015, 2016). Looking deep into the differences and into those factors that may contribute to low uptake rates might help inform future strategies to increase CR uptake (Al Quait \& Doherty, 2017; Karmali et al., 2014).

\section{Funding}

This work was supported by the British Heart Foundation (BHF) Cardiovascular Health Research Group which is supported by a grant from the BHF.

\section{Acknowledgements}

Thanks to the National Audit of Cardiac Rehabilitation (NACR) team in the Department of Health Sciences, University of York.

\section{Competing Interests Statement}

There are no conflicts of interest to declare.

\section{References}

Al Quait, A., \& Doherty, P. (2016). Does cardiac rehabilitation favour the young over the old? Open Heart, 3(2), e000450. http://doi.org/10.1136/openhrt-2016-000450

Al Quait, A., \& Doherty, P. (2017). Impact of age and gender on cardiac rehabilitation uptake in percutaneous coronary intervention patients. European Heart Journal, 38(suppl_1). http://doi.org/10.1093/eurheartj/ehx493.5972

Anderson, L., Oldridge, N., Thompson, D. R., Zwisler, A.-D., Rees, K., Martin, N., \& Taylor, R. S. (2016). Exercise-Based Cardiac Rehabilitation for Coronary Heart Disease. Journal of the American College of Cardiology, 67(1), 1-12. http://doi.org/10.1016/j.jacc.2015.10.044

BACPR. (2017). The BACPR Standards and Core Components for Cardiovascular Disease Prevention and Rehabilitation, 3rd ed. $\quad$ Retrieved 29 June 2017, from http://www.bacpr.com/resources/AC6_BACPRStandards\&CoreComponents2017.pdf

Bethell, H., Turner, S., EJ, F., \& Rose, L. (2000). The BACR database of cardiac rehabilitation units in the UK. Coronary Health Care, 4(2), 92-95. http://doi.org/http://dx.doi.org/10.1054/chec.2000.0072

Dalal, H. M., Doherty, P., \& Taylor, R. S. (2015). Cardiac rehabilitation. BMJ (Clinical Research Ed.), 351(sep29_11), h5000. http://doi.org/10.1136/bmj.h5000

Didier, J., Bigand, E., Vinter, A., \& Boucheix, J. (2010). Rethinking Physical and Rehabilitation Medicine. Springer. Retrieved from http://link.springer.com/content/pdf/10.1007/978-2-8178-0034-9.pdf

Doherty, P., \& Lewin, R. (2012). The RAMIT trial, a pragmatic RCT of cardiac rehabilitation versus usual care: what does it tell us? Heart, 98(8), 605-606. http://doi.org/10.1136/heartjnl-2012-301728

England, P., \& Guideline, C. (2014). Factsheet : Increase uptake of cardiac rehabilitation for people with coronary artery disease and following acute heart failure. Retrieved from https://www.england.nhs.uk/wp-content/uploads/2014/02/pm-fs-3-10.pdf

Groden, B. M., Semple, T., \& Shaw, G. B. (1971). Cardiac rehabilitation in Britain (1970). British Heart Journal, 33(5), 756-8. Retrieved from http://www.pubmedcentral.nih.gov/articlerender.fcgi?artid=487247\&tool=pmcentrez\&rendertype=abstract 
Humphrey, R., Guazzi, M., \& Niebauer, J. (2014). Cardiac rehabilitation in Europe. Progress in Cardiovascular Diseases, 56(5), 551-556. http://doi.org/10.1016/j.pcad.2013.08.004

Karmali, K. N., Davies, P., Taylor, F., Beswick, A., Martin, N., \& Ebrahim, S. (2014). Promoting patient uptake and adherence in cardiac rehabilitation. The Cochrane Database of Systematic Reviews, 6(2), CD007131. http://doi.org/10.1002/14651858.CD007131.pub3

Lavie, C. J., \& Milani, R. V. (2006). Adverse psychological and coronary risk profiles in young patients with coronary artery disease and benefits of formal cardiac rehabilitation. Archives of Internal Medicine, 166(17), 1878-1883. http://doi.org/10.1001/archinte.166.17.1878

Lawler, P. R., Filion, K. B., \& Eisenberg, M. J. (2011). Efficacy of exercise-based cardiac rehabilitation post-myocardial infarction: A systematic review and meta-analysis of randomized controlled trials. American Heart Journal, 162(4), 571-584.e2. http://doi.org/10.1016/j.ahj.2011.07.017

Lear, S. A., \& Ignaszewski, A. (2001). Cardiac rehabilitation: a comprehensive review. Current Controlled Trials in Cardiovascular Medicine, 2(5), 221-232. http://doi.org/10.1186/cvm-2-5-221

Linden, W., Stossel, C., \& Maurice, J. (1996). Psychosocial interventions for patients with coronary artery disease: a meta-analysis. Archives of Internal Medicine, 156(7), 745-52. http://doi.org/10.1001/archinte.1996.00440070065008

Mampuya, W. M. (2012). Cardiac rehabilitation past, present and future: an overview. Cardiovascular Diagnosis and Therapy, 2(1), 38-49. http://doi.org/10.3978/j.issn.2223-3652.2012.01.02

McGavock, J. M., Hastings, J. L., Snell, P. G., McGuire, D. K., Pacini, E. L., Levine, B. D., \& Mitchell, J. H. (2009). A forty-year follow-up of the Dallas Bed Rest and Training Study: The effect of age on the cardiovascular response to exercise in men. Journals of Gerontology - Series A Biological Sciences and Medical Sciences, 64(2), 293-298. http://doi.org/10.1093/gerona/gln025

Milani, R. V., Lavie, C. J., Mehra, M. R., \& Ventura, H. O. (2011). Impact of exercise training and depression on survival in heart failure due to coronary heart disease. American Journal of Cardiology, 107(1), 64-68. http://doi.org/10.1016/j.amjcard.2010.08.047

Montalescot, G., Sechtem, U., Achenbach, S., Andreotti, F., Arden, C., Budaj, A., .. Yildirir, A. (2013). 2013 ESC guidelines on the management of stable coronary artery disease. European Heart Journal, 34(38), 2949-3003. http://doi.org/10.1093/eurheartj/eht296

MORRIS, J. N., \& HEADY, J. A. (1953). Mortality in relation to the physical activity of work: a preliminary note on experience in middle age. British Journal of Industrial Medicine, 10(4), 245-54. Retrieved from http://www.pubmedcentral.nih.gov/articlerender.fcgi?artid=1037497\&tool=pmcentrez\&rendertype=abstrac $\mathrm{t}$

NACR. (2007). The National Audit of Cardiac Rehabilitation Annual Statistical Report 2007. York. Retrieved from http://www.cardiacrehabilitation.org.uk/docs/2007.pdf

NACR. (2014). The National Audit of Cardiac Rehabilitation- Annual statistical report 2014. York. Retrieved from http://www.cardiacrehabilitation.org.uk/docs/2007.pdf

NACR. (2015). The National Audit of Cardiac Rehabilitation- Annual statistical report 2014. Retrieved from http://www.cardiacrehabilitation.org.uk/docs/2007.pdf

NACR. (2016). the National Audit of Cardiac Rehabilitation Annual Statistical Report 2016. Retrieved from http://www.cardiacrehabilitation.org.uk/docs/BHF_NACR_Report_2016.pdf

Pavy, B., Iliou, M. C., Meurin, P., Tabet, J.-Y., \& Corone, S. (2006). Safety of exercise training for cardiac patients: results of the French registry of complications during cardiac rehabilitation. Archives of Internal Medicine, 166(21), 2329-34. http://doi.org/10.1001/archinte.166.21.2329

Rauch, B., Davos, C. H., Doherty, P., Saure, D., Metzendorf, M.-I., Salzwedel, A., ... Schmid, J.-P. (2016). The prognostic effect of cardiac rehabilitation in the era of acute revascularisation and statin therapy: A systematic review and meta-analysis of randomized and non-randomized studies - The Cardiac Rehabilitation Outcome Study (CROS). European Journal of Preventive Cardiology, 23(18), 1914-1939. http://doi.org/10.1177/2047487316671181

Sagar, V. A., Davies, E. J., Briscoe, S., Coats, A. J. S., Dalal, H. M., Lough, F., ... Taylor, R. S. (2015). Exercise-based rehabilitation for heart failure: systematic review and meta-analysis. Open Heart, 2(1), 
e000163. http://doi.org/10.1136/openhrt-2014-000163

Thomas, R. J., King, M., Lui, K., Oldridge, N., Pia, I. L., \& Spertus, J. (2010). AACVPR/ACCF/AHA 2010 update: Performance measures on cardiac rehabilitation for referral to cardiac rehabilitation/secondary prevention services. Journal of the American College of Cardiology, 56(14), 1159-1167. http://doi.org/10.1016/j.jacc.2010.06.006

Thompson, D. R. (1998). The development of a programme of research in cardiac rehabilitation. International Journal of Nursing Studies, 35((199X) 72-78), 72-78. Retrieved from http://ac.els-cdn.com/S0020748998000042/1-s2.0-S0020748998000042-main.pdf?_tid=2041d07c-6873-11 e7-8c14-00000aab0f01\&acdnat=1500023113_11276990451435a173be5849c2b6c698

Thompson, D. R., Bowman, G. S., Kitson, A. L., de Bono, D. P., \& Hopkins, A. (1996). Cardiac rehabilitation in the United Kingdom: guidelines and audit standards. National Institute for Nursing, the British Cardiac Society and the Royal College of Physicians of London. Heart, 75(1), 89-93. http://doi.org/10.1136/hrt.75.1.89

Thompson, P. D., Franklin, B. A., Balady, G. J., Blair, S. N., Corrado, D., Estes, N. A. M., ... Costa, F. (2007). Exercise and acute cardiovascular events: Placing the risks into perspective a scientific statement from the American Heart Association Council on Nutrition, Physical Activity, and Metabolism and the Council on $\begin{array}{llll}\text { Clinical Cardiology. } & \text { Circulation, } & \text { 2358-2368. }\end{array}$ http://doi.org/10.1161/CIRCULATIONAHA.107.181485

Townsend, N., Bhatnagar, P., Wilkins, E., Wickramasinghe, K., \& Rayner, M. (2015). Cardiovascular Disease Statistics 2014. London.

Turk-Adawi, K. I., Oldridge, N. B., Tarima, S. S., Stason, W. B., \& Shepard, D. S. (2013). Cardiac rehabilitation patient and organizational factors: What keeps patients in programs? Journal of the American Heart Association, 2(5). http://doi.org/10.1161/JAHA.113.000418

West, R. R., Jones, D. A., \& Henderson, A. H. (2012). Rehabilitation after myocardial infarction trial (RAMIT): multi-centre randomised controlled trial of comprehensive cardiac rehabilitation in patients following acute myocardial infarction. Heart, 98(8), 637-644. http://doi.org/10.1136/heartjnl-2011-300302

WHO. (2014). WHO | Risk factors. Retrieved 7 July 2017, from http:/www.who.int/topics/risk_factors/en/

\section{Notes}

Note 1. Modified from (Dalal et al., 2015).

Note 2. Adapted version from the NACR website.

Note 3. Courtesy of BACPR 2017.

\section{Copyrights}

Copyright for this article is retained by the author(s), with first publication rights granted to the journal.

This is an open-access article distributed under the terms and conditions of the Creative Commons Attribution license (http://creativecommons.org/licenses/by/4.0/). 